\title{
Grandfather Paradox in Non-Quantum and Quantum Gravitation Theories
}

\author{
Oleg Yurjevich Kupervasser \\ Transist Video LLC, Skolkovo, Moscow, Russia \\ Email: olegkup@yahoo.com
}

Received 11 May 2014; revised 13 June 2014; accepted 21 June 2014

Copyright (C) 2014 by author and Scientific Research Publishing Inc.

This work is licensed under the Creative Commons Attribution International License (CC BY).

http://creativecommons.org/licenses/by/4.0/

(c) (i) Open Access

\begin{abstract}
In this paper we resolve the grandfather paradox in non-quantum and quantum gravitation theories for time travelling in a time wormhole. For macroscopic bodies, the main solution is alignment of the thermodynamic time arrows, resulting in the time traveller destroying. For microscopic bodies and for small probability cases of macroscopic bodies, the main solution is fracture of the time wormhole. As a result, multi-world system appears. These explanations are similar in non-quantum and quantum gravity. On the contrary, we can clarify some problems of quantum gravity by this consideration. "Indestructible finite gravitation interaction of an observer with an observable system (resulting in the time arrows alignment)" and "instability with respect to even infinitesimally small interaction in the gravitation theory" can resolve the wave function reduction paradox of quantum mechanics.
\end{abstract}

\section{Keywords}

Component, Formatting, Style, Styling, Insert

\section{Introduction}

In Einstein's general relativity theory, motion is reversible, similar to classical mechanics. Nevertheless, two important topological differences exist between general relativity and classical mechanics.

The first difference is the ambiguity in general relativity in theory. In general relativity, two various initial states can give infinitesimally close states after a finite time interval. This phenomenon occurs, for example, during the formation of a black hole (Figure 1) as a result of a collapse. Let us consider the inverse process that describes a white hole. In this process, the infinitesimally close initial states after a finite time interval can yield different final states, which means that an observer/environment can considerably affect the evolution of the states during the finite time interval even when the observer/environment interacts infinitesimally weakly with 


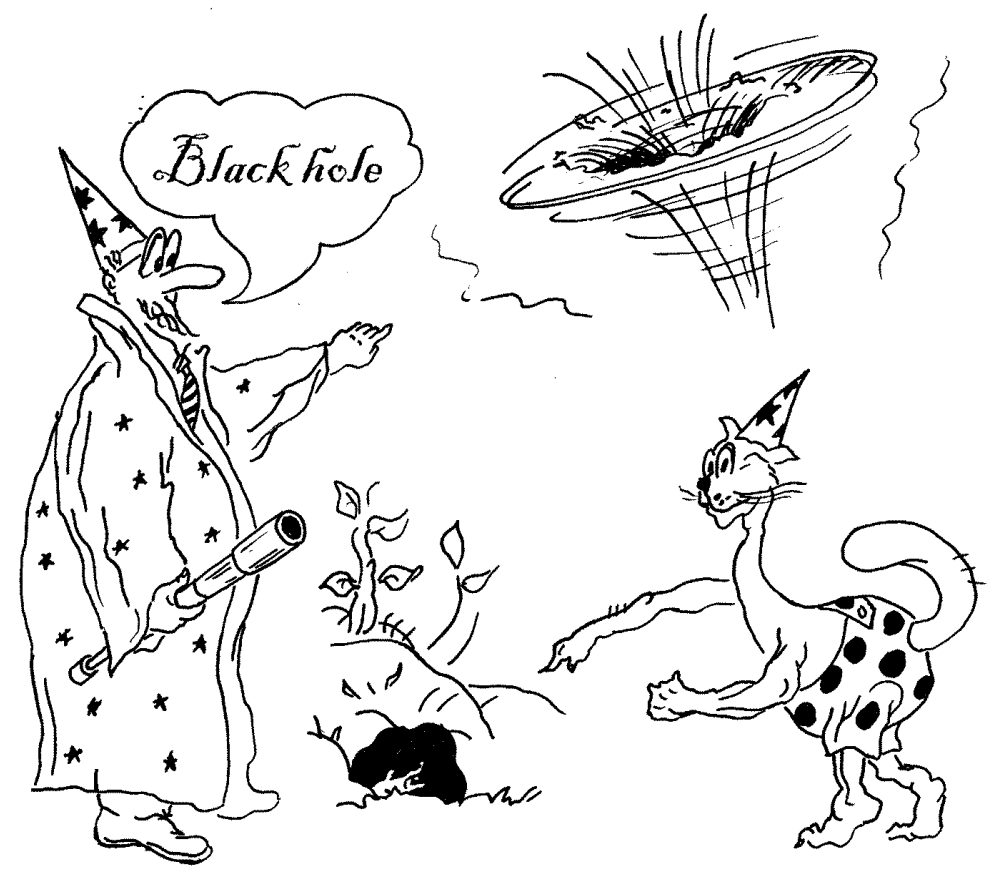

Figure 1. Black hole.

the white hole. As a result of this property, the law of increasing entropy turns out to be an exact law, not an FAPP law. Therefore, the entropy becomes a fundamental concept. There actually is a fundamental concept, such as the entropy of a black hole. Additionally, it is possible to explain the existence of this entropy using the perturbation created by the observer. Even now, this perturbation may be infinitesimally weak, unlike in classical mechanics. During the formation of a black hole, the entropy increases. Time reversion leads to the appearance of a white hole and to a decrease in entropy. The white hole cannot exist in reality because of the decrease in entropy. Decreases in entropy are prohibited in general relativity for the same reasons that they are prohibited in classical mechanics: the instability of the decreasing-entropy processes, which is much stronger in general relativity than that in classical mechanics. This instability results in the synchronisation of the time arrows of the white hole and of the observer/environment. The direction of the time arrow of the white hole changes to the opposite direction, coinciding with the time arrow of the observer/environment. The white hole transforms into a black hole. Note that a small interaction between the observer/environment and the observed system always exists in gravity theory, which is the gravity interaction. This interaction along with the above-described finite time instability produces unpredictability. This unpredictability cannot be prevented even theoretically. In contrast, in typical classical mechanics and quantum mechanics, unpredictability can be prevented in principle except for in some degenerated singular cases.

The second topological difference between classical mechanics and general relativity is the additional feedback between the final and initial states through a time-wise wormhole. Let us consider a wormhole [1] (see Figure 2), which is a paradoxical object of general relativity theory. Wormholes are geometric structures that connect two more-or-less flat regions of space-time. Arthur Charles Clarke writes [2] about the inanity of far interstellar travelling practically, even if it is technically possible. In fact, no effective communication between a star's civilisation makes sense if the time of signal interchange is longer than human life. However, wormholes can help resolve this problem [3]: "Should a traversable wormhole be found, it could be utilized in interstellar travel in the most obvious way. Suppose a traveler wants to fly from the Earth to Vega. One could think that the trip (there and back) will take at least 52 years (by the terrestrial clocks) even if she moves at a nearly light speed. But if there is a wormhole connecting the vicinities of the Earth and Vega she can take a short-cut by flying through it and thus make the round trip to Vega in (almost) no time... A possible way of creating hyperfast transport lies also in the use of traversable wormholes. Suppose that a traveler finds (or builds) a traversable wormhole with both mouths located near the Earth and suppose that she can move the mouths (see Figure 2) at will without serious damage to the geometry of the tunnel (which we take to be negligibly short), then she can 


\section{$2 \mathrm{D}$ model of wormhole}

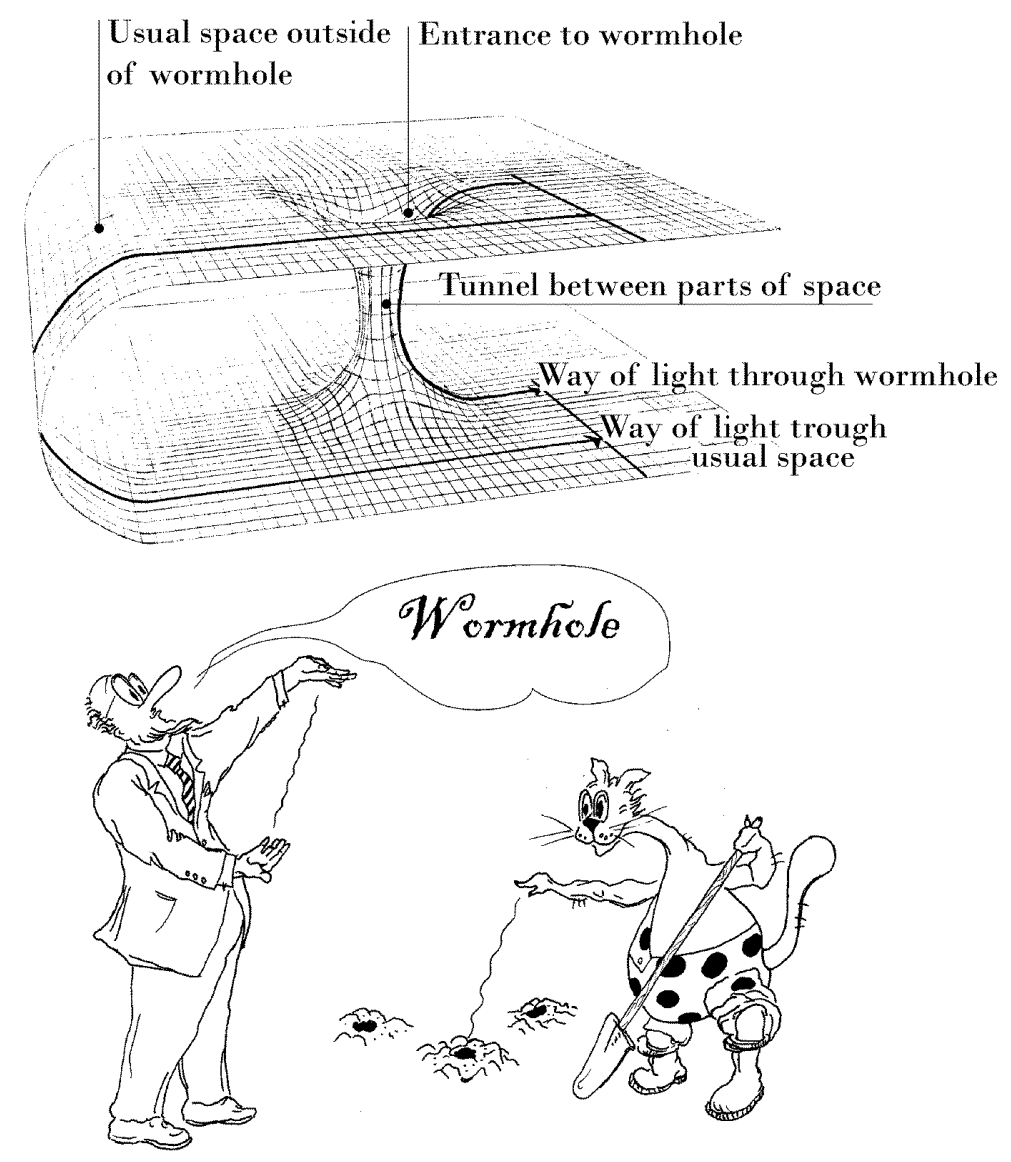

Figure 2. Wormhole.

fly to Vega taking one of the mouths with her. Moving (almost) at the speed of light she will reach Vega (almost) instantaneously by her clocks. In doing so she rests with respect to the Earth insofar as the distance is measured through the wormhole. Therefore, her clocks remain synchronous with those on the Earth as far as this fact is checked by experiments confined to the wormhole. So, if she returns through the wormhole she will arrive back to the Earth almost immediately after she will have left it". A wormhole can be used for human evacuation to another universe in the case of the destruction of our universe due to its accelerated expansion or shrinkage. Microscopic wormholes exist everywhere as fluctuations of the quantum vacuum. Nevertheless, to create real macroscopic wormholes from such small fluctuations, we need a significant amount of energy. Creating real macroscopic wormholes is a challenging problem for future technology.

We consider the Morris-Thorne wormhole [4]. Using a very simple procedure (we put one of the wormhole mouths on a spaceship, then the spaceship moves with a relativistic velocity over the closed loop and returns the mouth to its initial place), the wormhole that traverses space can be transformed into one that traverses time. After this transformation, the wormhole can be used as a time machine, leading to the paradox of the grandfather: a grandson can kill his grandfather, but it makes his birth impossible (Figure 3). For microscopic wormholes, the situation is different. If the initial conditions are compatible with travelling to the past through a wormhole, there are no reasons that can prevent it. However, this fact is not a solution to the grandfather paradox, which is a macroscopic, not microscopic, phenomenon.

\section{Non-Quantum Gravitation Theory}

This part considers the analysis of general relativity theory (the theory of gravitation) from the perspective of the 


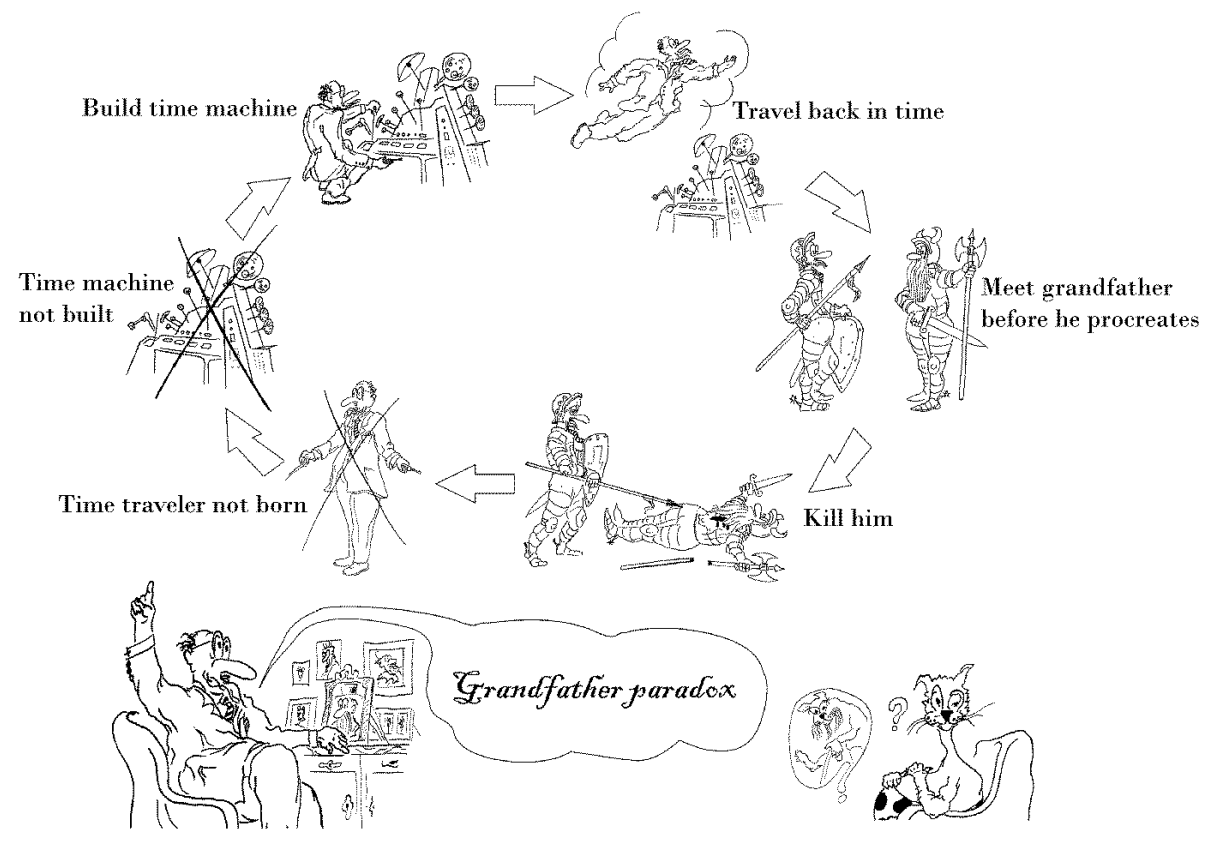

Figure 3. Grandfather paradox.

thermodynamic time arrow. Within this framework, the "paradox with the grandfather" for time travel "wormholes" is resolved.

We consider a thermodynamic time arrow [5] [6] (defined by the direction of the increase in entropy) within the limits of non-quantum relativistic gravitation theory. In classical Hamilton mechanics, any initial and final states are possible. Additionally, there is a one-to-one correspondence between these states. The situation is different with the relativistic theory of gravitation. There are topological singularities in space, which make a situation possible in which, for a finite time, different initial states yield identical final states, such as the collapse of black holes. However, having considered the inverse process in time, white holes, we obtain a situation in which a single initial state can yield a set of different final states for a finite time. There are also situations of other sorts in which non-arbitrary initial states are possible, including the case of "wormholes" through which time travel is possible. There is the necessity of self-consistency between the past and future, making some initial states impossible. "Wormholes" lead to the "paradox with the grandfather". The analysis of this situation from the perspective of the thermodynamic time arrow and the resolution of the above-defined paradox are the topic of this part.

\section{Time Wormhole}

Let us consider the paradoxical object of general relativity theory the time "wormhole" from the perspective of entropy [1]. First, we consider the most popular variant offered by Morris and Thorne [4]. Suppose we have a space wormhole with the extremities lying nearby. Using a very simple procedure (we place one of the extremities on a spaceship and move it with a speed close to the speed of light, and then, we return this extremity to the former place), this space wormhole can be converted into a time wormhole (a wormhole that traverses space into one that traverses time). This wormhole can be used as a time machine. Such a wormhole demands special exotic matter, which is necessary for conserving its equilibrium. However, there are models of a time machine without the exotic substance [7] [8]. Alternatively, the use of an electromagnetic field allows only a small amount of the matter to be used [9]. The use of such a time machine can lead to the well-known "paradox of the grandfather", in which the grandson, having returned to the past, kills his grandfather. How can this paradox be resolved?

From the physical perspective, the paradox of the grandfather means that not all of the initial states that exist before time machine formation are realisable. The introduction of additional feedback between the future and past, the time wormhole, makes these states impossible. Thus, we should either explain the non-reliability of 
such initial states ([10] and the thermodynamic arguments in this chapter) or suppose that the time "wormhole" is unstable, like a white hole, and changes easily.

Curiously enough, both of these explanations are true. However, for macroscopic wormholes, the first explanation has the highest priority. It would be very desirable to have a stable macroscopic topology of the space. The constraints on the initial states arise from the law of increasing entropy and the corresponding alignment of the thermodynamic time arrows that is related to the instability of states with opposite directions of time arrows [5] [6]. However, the macroscopic laws of thermodynamics are probabilistic. For a very small number of cases, they are not correct (large-scale fluctuations). For both these situations and microscopic wormholes, where the concept of a thermodynamic time arrow and thermodynamics laws are not applicable, the second explanation will have priority. This explanation is related to the extreme instability of the topology that is defined by the time machine [8]. We discussed above such a type of extreme instability for white holes. For macroscopic wormholes, the solution can be discovered using the law of increasing entropy. This law is ensured by the instability of the processes that have a decrease in entropy with respect to the Universe. This instability results in the alignment of the thermodynamic time arrows.

A space wormhole does not lead to a paradox. The objects immersed by its one extremity will go out the other extremity at later time. Thus, the objects from a more normalised low-entropy past occur in a less normalised high-entropy future. During the motion through the wormhole, the entropy of the travelling objects also increases: they transfer from a more normalised state into a less normalised state. Thus, the time arrows of the object travelling inside the wormhole and the time arrow of the world around the wormhole have the same directions. This situation is also true for travelling through the time wormhole from the past to the future.

However, for travelling from the future to the past, the time arrow directions of the traveller in the wormhole and of the world around the wormhole will be opposite [11]-[14]. In fact, the object travels from the less normalised future to the more normalised past, but its entropy increases instead of decreases! Thus, the thermodynamic time arrows of the Universe and of the traveller will have opposite directions. Such a process in which the entropy of the traveller decreases with respect to the Universe is unstable [5] [6]. Thus, the "memory about the past" of the traveller will be destroyed (and he may be destroyed completely), which will not allow him "to kill the grandfather".

Which mechanism for travelling in the wormhole ensures the alignment of the thermodynamic time arrows of the traveller and of the Universe? Both extremities of a "wormhole" are large bodies with a finite temperature. Both extremities under the second law of thermodynamics should inevitably radiate light, which partially penetrates into the wormhole. At the moment of the formation of a "time machine" (the transformation of a space wormhole into a time one), a closed light ray already appears between its extremities. Every time the ray spins in a circle, it becomes increasingly biased towards the violet part of the spectrum. Passing circle after circle, the rays lose their focal point; therefore, the energy does not get amplified and does not become infinite. The violet bias means that the history of a particle of light is finite and is defined by its coordinate time, despite the infinite number of circles [15]. This ray and other rays of light in the wormhole fluctuate. These rays also have a thermodynamic time arrow direction that coincides with the thermodynamic time arrow direction of the Universe. Due to the inevitable interaction with this radiation, the very unstable state of the traveller is destroyed. The state of the traveller is unstable because his thermodynamic time arrow is opposite to that of the Universe. The resulting destruction is sufficient to prevent the paradox of the grandfather.

Let us describe possible mechanism of the alignment of the thermodynamic time arrows and destroying a time traveller in a wormhole (see Figure 4, our explanation has some similarity with the analysis made in [16]). Feynman proposed an interpretation of the positron as an electron moving backward in time. A spaceman moves in a wormhole backward in time also (from T1 to T2). Therefore, similarly to the positron, we can consider the spaceman as a spaceman made from antimatter and moving forward in time. In the moment T1, the energy extracts. In the moment T2, the energy dissipates. In the moment T2, body and antibody appear. This appearance is an unstable process. The radiation in the wormhole destroys this process. As a result, some two clouds of particles and antiparticles appear instead of the spacemen. Anti-cloud annihilates with the spaceman in the moment T1 preventing the time travel.

"Free will" allows us to freely initiate only irreversible processes with an increase in entropy but not with a decrease in entropy. Thus, we cannot send an object from the future into the past. The process of the alignment of thermodynamic time arrows and the corresponding law of increasing entropy forbids the initial conditions that are necessary for the travelling of a macroscopic object into the past and the resulting "paradox of the 


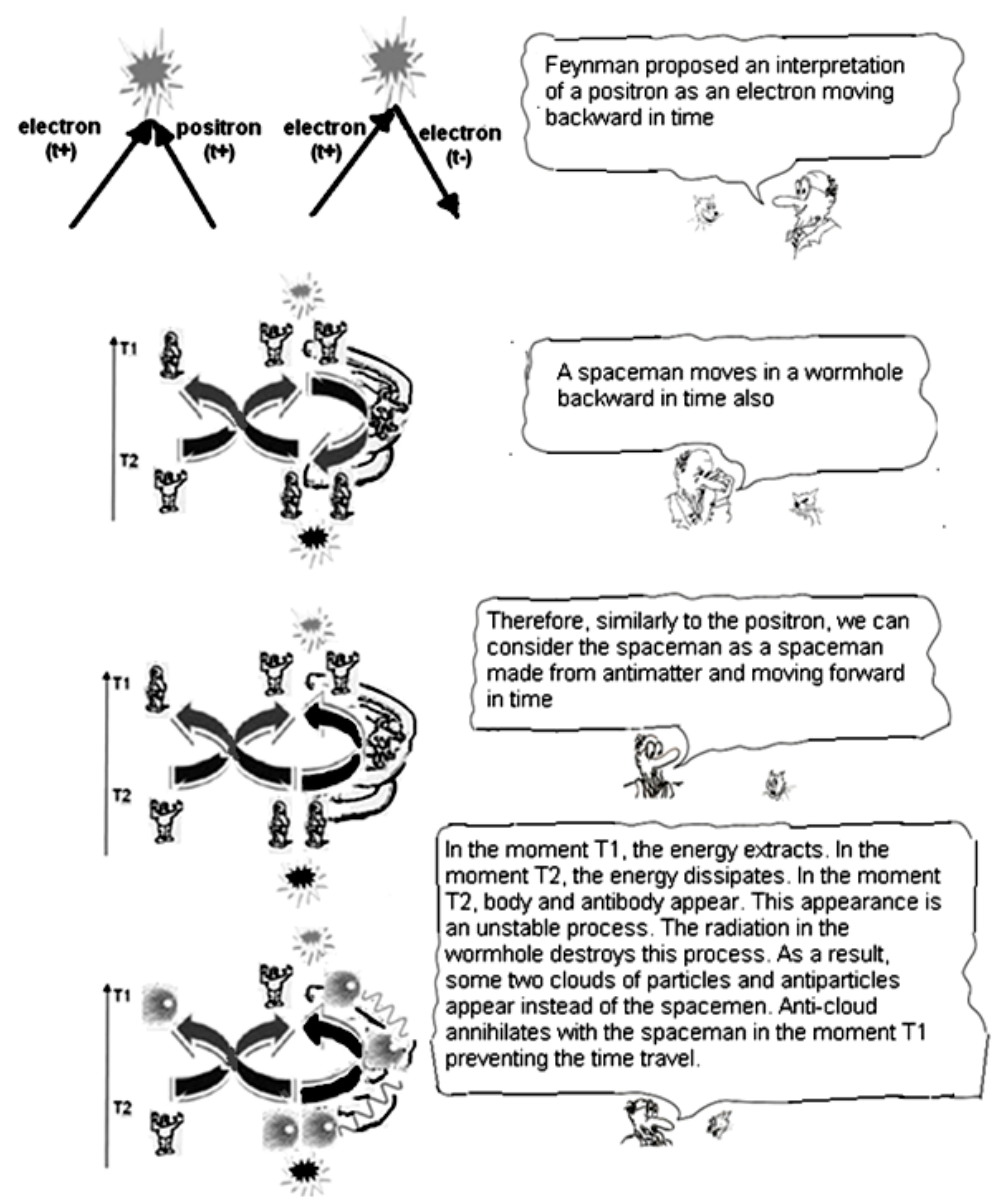

Figure 4. Resolution of the grandfather paradox.

grandfather”.

In paper [14], it is strictly mathematically proven that the thermodynamic time arrow cannot have an identical orientation with the coordinate time arrow during all travel over a closed time-like curve. The process of the alignment of the thermodynamic time arrows (related to the instability of the processes with entropy decrease) is this very physical mechanism, which actually ensures the law of increasing entropy.

The macroscopic laws of thermodynamics are probabilistic. For a very small number of cases, these laws do not work (large-scale fluctuations). For both these situations and microscopic systems, where thermodynamics laws are not applicable, the other explanation of the grandfather paradox will have priority. In this case, the time wormhole, like a white hole, appears unstable even with respect to infinitesimally weak perturbations from the gravitation of the travelling object. This instability can result in the wormhole's fracture and the prevention of the paradox, as is strictly proven in [8]. What are outcomes of the reorganisation of the space-time topology after the fracture of the time wormhole? The author of [8] writes:

"As we argue... non-uniqueness does not let the time travel paradoxes into general relativity-whatever happens in a causal region, a space-time always can evolve so that to avoid any paradoxes (at the sacrifice of the time machine at a pinch). The resulting space-times sometimes... curiously remind one of the many-world pictures”.

Let us formulate the following conclusion: for macroscopic processes, the instability of the processes with a decrease in entropy and the corresponding alignment of the thermodynamic time arrows make the existence of the initial conditions that allow travel to the past almost impossible. Thus, this instability prevents both wormhole fracture and the travelling of macroscopic bodies into the past that leads to the "paradox of the grandfather".

For the very improbable situations of macroscopic wormholes and for microscopic wormholes, wormhole 
fracture must occur. This fracture is the result of a remarkable property of general relativity theory-extreme instability: an infinitesimal external action (e.g., gravitation from a traveller) can produce a wormhole fracture for a finite time!

\section{Quantum Gravitation Theory}

This chapter considers the analysis of quantum gravitation theory from the perspective of the thermodynamic time arrow. Within this framework, the "paradox with the grandfather" for time travel "wormholes" is considered. This paper includes an analysis of quantum gravitation theory from the perspective of the thermodynamic time arrow [5] [6] [15]. Within this framework, the "paradox of the grandfather" for time "wormholes" is considered.

\section{Time Wormhole}

Let us consider the paradoxical object of general relativity theory the time "wormhole" from the perspective of entropy [1]. First, we consider the most popular variant offered by Morris and Thorne [4]. Suppose we have a space wormhole with the extremities lying nearby. Using a very simple procedure (we place one of the extremities on a spaceship and move it with a speed close to the speed of light, and then, we return this extremity to the former place), this space wormhole can be converted into a time wormhole (a wormhole that traverses space into one that traverses time), which can be used as a time machine. Such a wormhole demands special exotic matter, which is necessary for conserving its equilibrium. However, there are models of a time machine that enable the exotic substance to be disregarded [7] [8]. Using an electromagnetic field, a small amount of the matter can be eliminated [9]. The use of such a time machine can lead to the well-known "paradox of the grandfather", in which the grandson, having returned to the past, kills his grandfather. How can this paradox be resolved?

Let us consider the fact that the answer to this problem is given by the semi-classical theory of gravitation. Suppose that the macroscopic topology of the space that is related to the time machine is unchanged. At the moment of time machine formation (the transformation of the space wormhole into the time one), there is a closed light ray between its extremities. The energy of the light ray does not reach infinity, despite the infinite number of passes, because of the defocusing of the light [16]. Another situation, however, arises in the semi-classical theory with the radiation field of "vacuum fluctuations" [17]. After passing an infinite number of times through the wormhole and being summed with each other, these fluctuations reach an infinite energy, which will destroy any traveller.

However, the situation in quantum gravitation is different. Quantum fluctuations contain large energies when they arise over short distances. Therefore, it is possible to find a distance so small that the energy of the fluctuation will be sufficiently large to form a tiny black hole, and the horizon of this tiny black hole will have the same size as this small distance. Space-time is not capable of remaining homogeneous over such short distances. This mechanism ensures the natural "blocking" of singular fluctuation formation, restricting the fluctuations in their sizes: the "maximum energy in minimal sizes" [15].

The detailed calculations of quantum gravitation show [18] that this "blocking" of the formation of singular fluctuations provides a very small, but non-zero, probability for an unobstructed transition through a time "wormhole" for a macroscopic object. How can the "paradox of the grandfather" be prevented in this situation? Here, it is convenient to use the language of the multi-world interpretation of quantum mechanics. To prevent this paradox, the traveller should penetrate into the parallel world in which it can easily "kill the grandfather" without breaking the causality principle. Such a parallel world will interfere quantum-mechanically with the worlds of the "not killed grandfather", in which the observer was unsuccessful in transiting the time wormhole. However, the probability amplitude of such a world will be extremely small. Can the observer in the world where "the grandfather is not killed" discover the alternative world, at least in principle, using quantum correlations between the worlds? Similarly to the "paradox of the Schrödinger cat", he cannot do so for the same reasons as in conventional quantum mechanics [6]. The observation of the large effects of quantum correlations is impossible because of "observer memory erasing" [5] [6]. Penetration into the parallel world of quantum mechanics is experimentally indistinguishable from the time wormhole fracture and from penetration into the parallel world of general relativity theory [8]. This result means that from the perspective of the external real macroscopic observer, the situations in which the traveller has perished in the wormhole or has penetrated into "another world" are observationally indistinguishable. This situation is equivalent to when the traveller falls into a 
black hole. We do not know whether he is crushed in the singularity or has penetrated into "the other world" through the white hole [19]. (This difference is, however, observed and essential for the traveller. He will carry away all of these observations with himself into "the other world"). We see that the experimental difference between the quantum and semi-classical theories for macroscopic objects is not observed for the macroscopic observer, which is not travelling in the time wormhole.

\section{Conclusions}

We see that the informational paradox and the paradox of the grandfather are resolved in quantum gravitational theory similarly to in non-quantum general relativity theory. This resolution is realized through the consideration of the weak interaction of the systems with the real non-equilibrium macroscopic observer. Moreover, this approach (similar to in conventional quantum theory) enables the resolution of the wave packet reduction problem [5] [6]. However, this reduction in quantum gravitation becomes a fundamental property of the theory, unlike in the case of conventional quantum mechanics. This is result of

1) Indestructible finite gravitation interaction of an observer with an observable system;

2) Instability with respect to even infinitesimally small interaction in the gravitation theory.

\section{Acknowledgements}

I thank the painter Gukov Yury Yurjevich for his help in drawing the figures.

\section{References}

[1] Noyola, J.P. (2006) Relativity and Wormholes.

http://www.uta.edu/physics/main/resources/ug_seminars/papers/RelativityandWormholes.doc

[2] Clarke, A.C. (1962) Profiles of the Future. Harper \& Row, New York.

[3] Krasnikov, S.V. (2000) Toward a Traversable Wormhole. Space Technology and Application International Forum. http://arxiv.org/abs/gr-qc/0003092v1

[4] Morris, M. and Thorne, K. (1988) Wormholes in Spacetime and Their Use for Interstellar Travel: A Tool for Teaching General Relativity. American Journal of Physics, 56, 395-416. http://dx.doi.org/10.1119/1.15620

[5] Kupervasser, O., Nikolic, H. and Zlatić, V. (2012) Universal Arrow of Time. Foundations of Physics, 42, $1165-1185$. http://dx.doi.org/10.1007/s10701-012-9662-8

[6] Kupervasser, O. (2013) The Universal Arrow of Time Is a Key for the Solution of the Basic Physical Paradoxes. Electronic Journal of Theoretical Physics, 10, 39.

[7] Amos, O. (2005) A Class of Time-Machine Solutions with a Compact Vacuum Core. Physical Review Letters, 95, 021101-021104. http://dx.doi.org/10.1103/PhysRevLett.95.021101

[8] Krasnikov, S.V. (2002) Time Travel Paradox. Physical Review D, 65, 064013-064020. http://arxiv.org/abs/gr-qc/0109029 http://dx.doi.org/10.1103/PhysRevD.65.064013

[9] Novikov, I.D., Kardashev, N.S. and Shatskii, A.A. (2007) The Multicomponent Universe and the Astrophysics of Wormholes. Physics-Uspekhi, 177, 1017.

[10] Friedman, J., Morris, M., Novikov, I., Echeverria, F., Klinkhammer, G., Thorne, K. and Yurtsever, U. (1990) Cauchy Problem in Spacetimes with Closed Timelike Curves. Physical Review D, 42, 1915-1930. http://dx.doi.org/10.1103/PhysRevD.42.1915

[11] Zeh, H.D. (2007) The Physical Basis of the Direction of Time. Springer, Heidelberg.

[12] Zeh, H.D. (2005) Remarks on the Compatibility of Opposite Arrows of Time. Entropy, 7, 199-207. http://dx.doi.org/10.3390/e7040199

[13] Zeh, H.D. (2006) Remarks on the Compatibility of Opposite Arrows of Time II. Entropy, 8, 44-49. http://dx.doi.org/10.3390/e8010044

[14] Nikolic, H. (2006) Causal Paradoxes: A Conflict between Relativity and the Arrow of Time. Foundations of Physics Letters, 19, 259-267. http://dx.doi.org/10.1007/s10702-006-0516-5

[15] Hawking, S.W., Thorne, K.S., Novikov, I., Ferris, T., Lightman, A. and Price, R. (2002) The Future of Spacetime. Institute of Technology, Pasadena.

[16] Shulman, M.H. (2012) Is It Possible to Travel in Time? 
http://www.timeorigin21.narod.ru/eng_time/Is_it_possible_to_travel_in_time_eng.pdf

[17] Kim, S.W. and Thorne, K.S. (1991) Do Vacuum Fluctuations Prevent the Creation of Closed Timelike Curves? Physical Review D, 43, 3929. http://dx.doi.org/10.1103/PhysRevD.43.3929

[18] Cassidy, M.J. and Hawking, S.W. (1998) Models for Chronology Selection. Physical Review D, 57, $2372-2380$. http://dx.doi.org/10.1103/PhysRevD.57.2372

[19] Popławski, N.J. (2010) Radial Motion into an Einstein-Rosen Bridge. Physics Letters B, 687, 110-113. http://dx.doi.org/10.1016/j.physletb.2010.03.029 
Scientific Research Publishing (SCIRP) is one of the largest Open Access journal publishers. It is currently publishing more than 200 open access, online, peer-reviewed journals covering a wide range of academic disciplines. SCIRP serves the worldwide academic communities and contributes to the progress and application of science with its publication.

Other selected journals from SCIRP are listed as below. Submit your manuscript to us via either submit@scirp.org or Online Submission Portal.
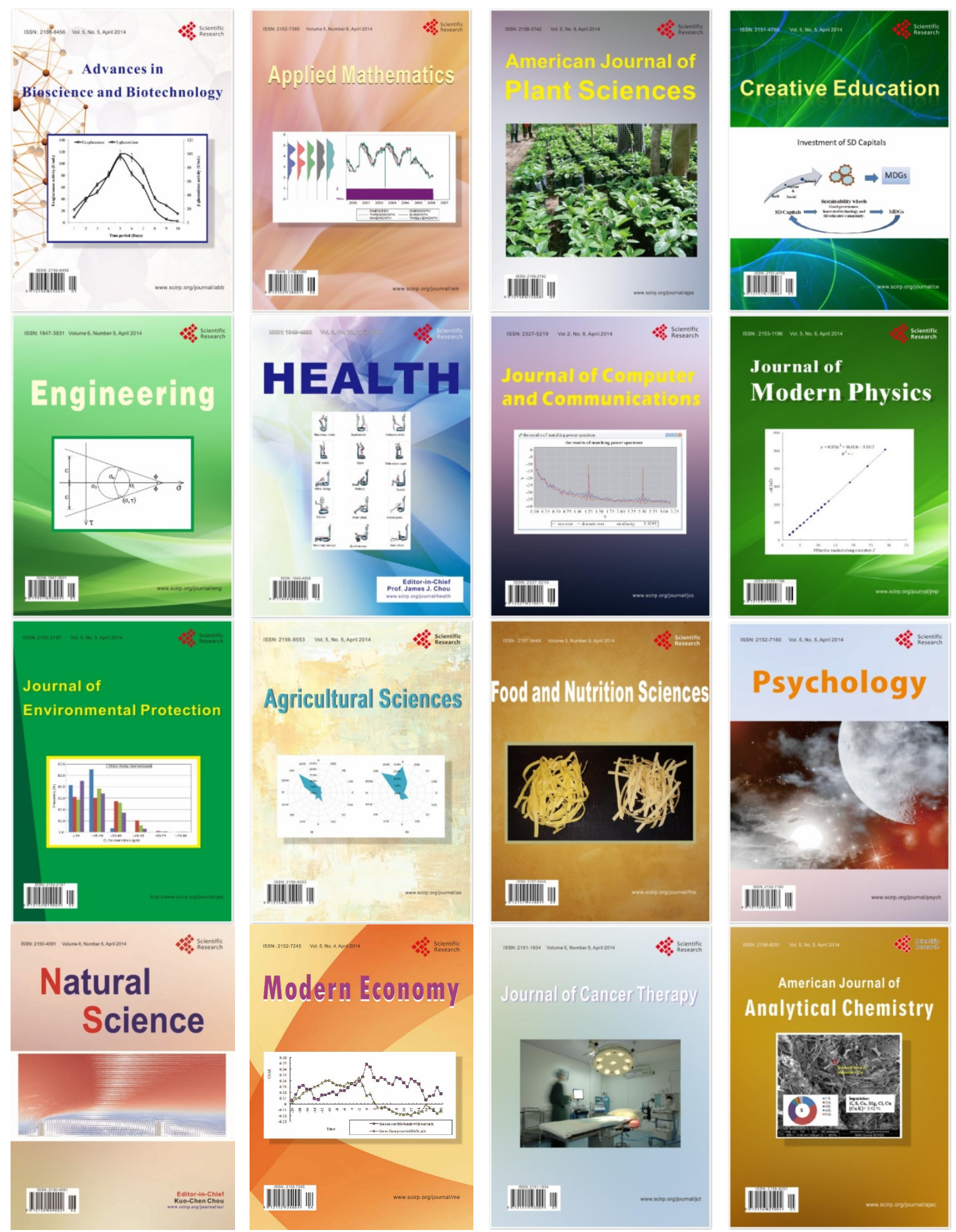